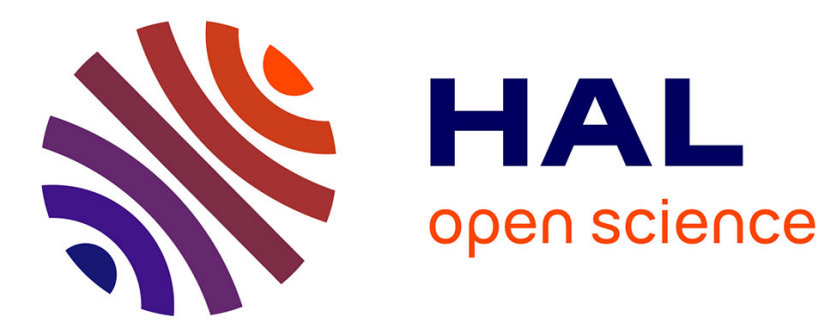

\title{
Some Properties of Interpolations Using Mathematical Morphology
}

Aditya Challa, Sravan Danda, B S Daya Sagar, Laurent Najman

\section{To cite this version:}

Aditya Challa, Sravan Danda, B S Daya Sagar, Laurent Najman. Some Properties of Interpolations Using Mathematical Morphology. IEEE Transactions on Image Processing, 2018, 27 (4), pp.2038 2048. 10.1109/TIP.2018.2791566 . hal-01484894v3

\section{HAL Id: hal-01484894 \\ https://hal.science/hal-01484894v3}

Submitted on 9 Jan 2018

HAL is a multi-disciplinary open access archive for the deposit and dissemination of scientific research documents, whether they are published or not. The documents may come from teaching and research institutions in France or abroad, or from public or private research centers.
L'archive ouverte pluridisciplinaire HAL, est destinée au dépôt et à la diffusion de documents scientifiques de niveau recherche, publiés ou non, émanant des établissements d'enseignement et de recherche français ou étrangers, des laboratoires publics ou privés. 


\title{
Some Properties of Interpolations Using Mathematical Morphology
}

\author{
Aditya Challa, Student Member, IEEE, Sravan Danda, Student Member, IEEE B. S. Daya Sagar, Senior \\ Member, IEEE and Laurent Najman, Senior Member, IEEE
}

\begin{abstract}
The problem of interpolation of images is defined as - given two images at time $t=0$ and $t=T$, one must find the series of images for the intermediate time. This problem is not well posed, in the sense that without further constraints, there are many possible solutions. The solution is thus usually dictated by the choice of the constraints/assumptions, which in turn relies on the domain of application. In this article we follow the approach of obtaining a solution to the interpolation problem using the operators from Mathematical Morphology (MM). These operators have an advantage of preserving structures since the operators are defined on sets. In this work we explore the solutions obtained using MM, and provide several results along with proofs which corroborates the validity of the assumptions, provide links among existing methods and intuition about them. We also summarize few possible extensions and prospective problems of current interest.
\end{abstract}

Index Terms-Image Interpolation, Mathematical Morphology, Morphological Interpolation.

\section{INTRODUCTION}

$\mathbf{T}$ HE problem of image interpolation can be stated as given two images $I_{0}$ (source) and $I_{1}$ (target), find the series of images $\left\{Z_{\alpha}, \alpha \in[0,1]\right\}$ such that $Z_{0}=I_{0}$ and $Z_{1}=I_{1}$. Image interpolation problem requires the answers to be 'visually appealing' which is very hard to characterize rigorously. For instance in figure 1, let the source image be as in (a) and target image be as in (b). A simple linear interpolation $\left(0.5 * I_{0}+0.5 * I_{1}\right)$ would result in the one as obtained in (c). However, one intuitively expects that - the 'structure' to change from the circle to square gradually.

Image interpolation problem is also referred to as image morphing which is widely used for special effects and animation [18], [39], [37]. In this work, the aim is to analyze the problem from the perspective of remote sensing and geoscience and, as described in section II, the nature of the solutions is very different from that of image morphing. Several possible applications exist for such a solution. For example, a satellite maps the surface at regular intervals and one might be interested in visualizing the intermediate states. Apart from this, the problem of image interpolation is of

Manuscript received March 26, 2017; revised August 21, 2017; accepted xxxx xx, xxxx. B. S. D. Sagar would like to acknowledge the support received from the Indian Space Research Organization (ISRO) with the grant number ISRO/SSPO/Ch-1/2016-17.

Aditya Challa (aditya.challa.20@gmail.com), Sravan Danda (sravandanda@gmail.com) and B.S.Daya Sagar (bsdsagar@isibang.ac.in) are with the Systems Science and Informatics Unit, Indian Statistical Institute - BangaloreCenter, Bangalore, 560059,India.

Laurent Najman (laurent.najman@esiee.fr) is with Université Paris-Est, LIGM, Equipe A3SI, ESIEE, Paris, France. interest to the Geographic Information Science community [26].

Note that this problem is different from the usual interpolation problem - given a function values at a few points, find the value of the function at the intermediate points [19], [36]. Since the techniques used in this article are developed under the branch of 'morphological interpolation', we refer to the former problem as the image interpolation problem and the latter problem as the image scaling problem. The image scaling problem requires estimating the pixel values in between the existing pixels, which would in turn increase the resolution of the image. In contrast, the resolution of the images in the interpolation problem does not change. We remark that few of the techniques used for one problem can be used for other as well (with slight modification), but the end objective of the problem are inherently different. We also remark that morphological techniques have been used for image upscaling [17].

The solution to the image interpolation problem can be approached from various starting points. In this work, the main interest is in analyzing the solution obtained using operators from Mathematical Morphology (MM). MM is a theory of non-linear operators on images introduced by Georges Matheron and Jean Serra in the late 1970s [32]. These operators are famous for preserving the 'structure' as the operators are defined on sets instead of pixels. This makes the subject of morphological operators a good candidate to deal with the problem of image interpolation

Different solutions to the problem of image interpolation via MM operators are proposed in several works [23], [30]. One of the aims of this article is to provide a consolidated theoretical review of the earlier methods and claim that MM operators are suitable for interpolation. Several extensions and prospective problems for future work are also discussed. The main contributions of this article are -

- Systematic review of the ideas presented in the existing literature, consolidated theoretically. Specifically, the propositions 1 - 6 provide better intuition and understanding of the assumptions made and methods discussed. To the best of our knowledge, all properties presented in this article are novel.

- Few simple simulated examples which discuss the pros and cons of the methods.

- Provide a platform for various extensions and future work.

The methods described in this article are not the only possible solutions to the interpolation problem [5], [34], [23]. 
Also, we concentrate on binary and greyscale interpolations but these techniques can also be extended to color images as well [9], [1].

The outline of the paper is as follows - In section II, we briefly describe various MM operators and other definitions required for the remaining portion of the article. In section III, we discuss one approach to interpolates using MM. In section IV, we reduce the problem of interpolation to the problem of constructing the median by a series of simplifications. We offer several results justifying the assumptions. In section V, we discuss yet another approach to interpolates, and identify the links with Serra's median (definition 3). We then briefly review the interpolation of greyscale images with focus on flat vs non-flat structuring elements in section VI. In section VII, we provide several problems for future research and briefly describe each of these problems.

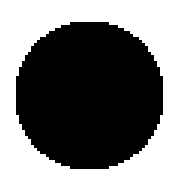

(a)

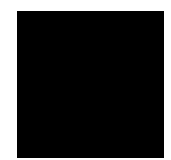

(b)

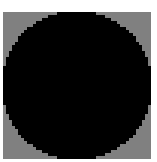

(c)
Fig. 1. (a) Source Image. (b) Target Image. (c) Linear interpolation between (a) and (b).

\section{REVIEW OF MORPHOLOGICAL OPERATORS}

In this section we introduce the basic operators of Mathematical Morphology (MM), recall the definitions and setup the notation as required by the rest of the article [29], [24], [22], [28].

Loosely speaking, the basic MM operators - dilation, erosion, opening and closing, are defined on binary images and can be extended to greyscale images. This is achieved on the abstract level by defining the operators on abstract structures called Complete Lattices. However, for the purposes of this article, we only review the basic operators on binary and greyscale images. Interested readers can refer to [29], [24] for theoretical details on MM operators and [3], [7] for details about lattices.

\section{A. Binary Images}

A binary image is a map, $I: E \rightarrow\{0,1\}$. $E$ is called the domain of definition and is usually taken as $\mathbb{R}^{2}$ or $\mathbb{Z}^{2}$. Here $\mathbb{R}$ refers to the real line and $\mathbb{Z}$ denotes the set of integers. For most of theoretical aspects we consider $E=\mathbb{R}^{2}$. Note that, practically, images are restricted to a finite domain. However results, henceforth, are stated on infinite domain and hold true for finite domains as well. This distinction is blurred for the rest of the article for pedagogical reasons.

An equivalent way of characterizing the binary image is to look at sets $\{x \in E \mid I(x)=1\}$. Such sets belong to the space of $\mathbb{P}(E)$, where $\mathbb{P}(E)$ is the power set - set of all possible subsets of $E$. Basic MM operators on binary are maps from $\mathbb{P}(E) \rightarrow \mathbb{P}(E)$. To define these operators one needs another set, called structuring element, with a defined origin.

There are several ways to look at the structuring element. Theoretically, a structuring element is the set to which the set $\{0\}$ maps to. Then using the assumption - Invariance to translation, we can extend the mapping to all unit sets $\{x\}$. Then using the assumption - Invariance to supremum gives us the dilation and using the assumption -invariance to infimum gives us erosion. For the remaining part of the paper it is assumed that $B$ denotes a unit disk with origin at the center. Accordingly, $\lambda B$ indicates the disk with radius $\lambda$.

1) Dilation: The first MM operator we discuss is that of Morphological Dilation, or simply Dilation, $\delta_{B}($.$) . Recall that$ it is a map from $\mathbb{P}(E)$ to itself. Thus we have

$$
\delta_{B}(X)=X \oplus B=\bigcup_{b \in B} X_{b}=\left\{x \in E \mid B_{x} \cap X \neq \emptyset\right\},
$$

where $X \oplus B$ is usual Minkowski addition and $X_{b}$ is the set $X$ translated by $b$.

2) Erosion: Morphological Erosion, or simply Erosion, $\epsilon_{B}($.$) with respect to the structuring element B$ is defined as

$$
\epsilon_{B}(X)=X \ominus \hat{B}=\bigcap_{b \in B} X_{-b}=\left\{x \in E \mid B_{x} \subseteq X\right\},
$$

where $X \ominus B$ is usual Minkowski subtraction. When the dilations and erosions are restricted to a domain, they are called Geodesic Dilations and Geodesic Erosions respectively [33]. Assume that we have two sets $X \subset Y$. The geodesic dilation and geodesic erosion are respectively defined by

$$
\begin{aligned}
\Delta_{Y, B}(X) & =\delta_{B}(X) \cap Y \\
\mathcal{E}_{X, B}(Y) & =\epsilon_{B}(Y) \cup X
\end{aligned}
$$

\section{B. Greyscale Images}

A greyscale image is defined as a function $I: \mathbb{R}^{2} \rightarrow \overline{\mathbb{R}}$, where $\overline{\mathbb{R}}=\mathbb{R} \cup\{-\infty, \infty\}$. An equivalent way of looking at the greyscale image is using the umbra [24] defined as

$$
U(I)=\{(x, t) \mid I(x) \leq t\}
$$

Note that $U(I) \subset \mathbb{R}^{3}$. Using these sets, the definitions of dilation and erosion for binary images can be extended to greyscale images as well. The main distinction being how the structuring element is extended from $\mathbb{R}^{2}$ to $\mathbb{R}^{3}$.

The most important point to note is that - all dimensions are not created equal. Observe that two dimensions correspond to the spatial co-ordinates while the third corresponds to the greyscale value. The spatial co-ordinates can be handled the same way as before. The greyscale value on the other hand, can be handled in two ways - with/without changing the maximum greyscale value in the image. This results in non-flat and flat structuring elements respectively.

1) Flat Structuring Elements: Given a set $B \subset \mathbb{R}^{2}$, a flat structuring element is defined as

$$
g(x)= \begin{cases}0 & x \in B \\ -\infty & x \notin B\end{cases}
$$


2) Non-Flat Structuring Elements: Given a set $B \subset \mathbb{R}^{2}$, a non-flat structuring element is defined as

$$
g(x)= \begin{cases}i_{x} & x \in B \\ -\infty & x \notin B\end{cases}
$$

where $i_{x}$ can take any value in $\mathbb{R}$.

3) Greyscale Operators: Greyscale Morphological Dilation with respect to the structuring element $g(x), \delta_{g}($.$) is defined$ as

$$
\delta_{g}(f)(x)=\sup \{f(h)+g(x-h) \mid h \in E\}
$$

Greyscale Morphological Erosion with respect to the structuring element $g(x), \epsilon_{g}($.$) is defined as$

$$
\epsilon_{g}(f)(x)=\inf \{f(h)-g(x-h) \mid h \in E\}
$$

The importance of the distinction between flat and nonflat structuring elements and greyscale operators are further discussed in section VI. Unless otherwise mentioned, in what follows the dilation and erosion operators refer to operators on binary images.

\section{Hausdorff Distances}

Another concept used in the remaining portion of the article is that of distance between sets. There are various distances which can be defined on two sets. Dilation distance, $\bar{d}(X, Y)$ is defined as $\inf \left\{\lambda \mid \delta_{\lambda B}(X) \supseteq Y\right\}$. Hausdorff Dilation distance is defined as $d(X, Y)=\sup \{\bar{d}(X, Y), \bar{d}(Y, X)\}$. The following are the list of properties which follow from the definition -

1) $d(X, Y)=d(Y, X)$

2) $d(X, Y)=0$ if and only if $X=Y$.

3) $d(X, Y) \leq d(X, Z)+d(Y, Z)$.

4) If we have that $X \subseteq Y$, then $d(X, Y)=\bar{d}(X, Y)$

We can also define the dual distance operator, Erosion distance as $\bar{e}(X, Y)$ is defined as $\inf \left\{\lambda \mid \epsilon_{\lambda B}(X) \subseteq Y\right\}$. And the Hausdorff Erosion distance is defined as $e(X, Y)=$ $\sup \{\bar{e}(X, Y), \bar{e}(Y, X)\}$. Note that, the convention that $\emptyset \subset X$ for all sets $X$ is followed here.

\section{Influence Zones}

If $Z_{1}$ and $Z_{2}$ are two sets in $\mathbb{R}^{2}$, then the influence zone of $Z_{1}$ with respect to $Z_{2}$ is defined as

$$
I Z\left(Z_{1} \mid Z_{2}\right)=\left\{x: \bar{d}\left(Z_{1}, x\right)<\bar{d}\left(Z_{2}, x\right)\right\}
$$

Similarly, influence zone of $Z_{2}$ with respect to $Z_{1}$ is defined as

$$
I Z\left(Z_{2} \mid Z_{1}\right)=\left\{x: \bar{d}\left(Z_{2}, x\right)<\bar{d}\left(Z_{1}, x\right)\right\}
$$

One can give a simpler characterization for the influence zone. Fix a point $x$. Now $x \in I Z\left(Z_{1} \mid Z_{2}\right)$ if and only if we have that $x \in Z_{1} \oplus \lambda B$ and $x \notin Z_{2} \oplus \lambda B$. That is $x \in$ $\left(Z_{2} \oplus \lambda B\right)^{c}$. Thus we have

$$
I Z\left(Z_{1} \mid Z_{2}\right)=\bigcup_{\lambda \geq 0}\left(Z_{1} \oplus \lambda B\right) \cap\left(Z_{2} \oplus \lambda B\right)^{c}
$$

We can also define the Skeleton by Influence Zone (SKIZ) as

$$
\operatorname{SKIZ}\left(Z_{1}, Z_{2}\right)=\left\{x: \bar{d}\left(Z_{2}, x\right)=\bar{d}\left(Z_{1}, x\right)\right\}
$$

Remark : As stated before, there exists several possible solutions to an interpolation problem. To obtain a unique solution, one needs to place constraints on the solution set. Such constraints are usually dictated by the domain of application. One of the areas of application of morphological interpolation is that of geo-spatial variables [27].

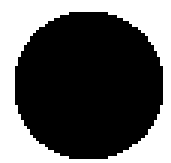

(a)

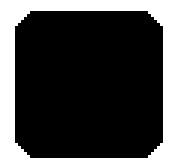

(d)

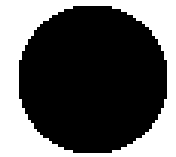

(b)

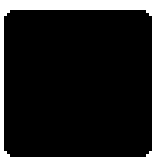

(e)

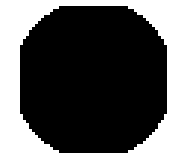

(c)

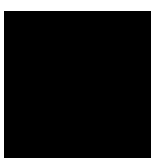

(f)
Fig. 2. (a) Source Image. (b) - (e) Geodesic Dilation. (f) Target Image. Recall that MM operators act by changing the structure of the set. The images in this figure are generated using geodesic dilation. Contrasting them with that of figure 1 we find that interpolates in this figure modify the structure while interpolation in figure 1 modifies the grey values. Hence interpolates in this figure provide a better solution. This is especially suitable for the case of geospatial variables since MM operators simulate several geophysical processes.

\section{FIRST ATTEMPTS}

In this section we review the two methods described in [30]. Recall that we are interested in constraints so that we can pick uniquely the set of interpolates between the two images. One such constraint is given by Hausdorff distances. That is, one picks shortest path between source and target as dictated by Hausdorff distances.

Rigorously speaking, consider the space $\mathbb{P}(E)$, with the metric given by Hausdorff distance. If $X$ and $Y$ are two sets, then we shall be interested in shortest path between $X$ and $Y$ in this space. A shortest path given by the First Hausdorff Interpolates is defined below.

Definition 1 (First Hausdorff Interpolates). Let $X$ and $Y$ be two sets. Let $\rho=d(X, Y)$ (Hausdorff distance). Let $B$ denote the disk structuring element with radius 1 . Then the first Hausdorff interpolates, $\left\{Z_{r}: \alpha \in[0,1]\right\}$ is defined as

$$
Z_{\alpha}=\delta_{\alpha \rho B}(X) \bigcap \delta_{(1-\alpha) \rho B}(Y)
$$

The interpolates defined in definition 1 satisfy

$$
d\left(Z_{\alpha}, X\right)=(\alpha) \rho \quad d\left(Z_{\alpha}, Y\right)=(1-\alpha) \rho
$$


The proof of this can be found in [30]. The above relation implies that the first Hausdorff interpolates falls on the shortest path between the sets $X$ and $Y$.

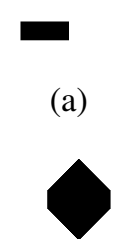

(e)

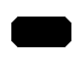

(b)

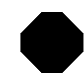

(f)

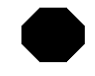

(c)

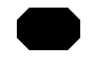

(g)

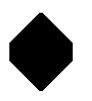

(d)

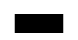

(h)
Fig. 3. First Hausdorff Interpolates. (a) Source Image. (b) - (g) First Hausdorff Interpolates. (h) Target Image. Note that the target image is a simple translation of the object in source image.

An example of the first Hausdorff interpolates is given in figure 3. It is observed that these interpolates even though theoretically sound, suffer from the problem of 'thick' interpolates as illustrated in figure 3 . There are two ways around this problem - 1) Observe that the sets $X$ and $Y$ are simple translations of each other. Thus negating the affine transformations might reduce this effect. 2) A simpler solution is to restrict the interpolates to the convex hull of sets $X$ and $Y$. This also reduces the problem of 'thick' interpolates.

Definition 2 (Second Hausdorff Interpolates). Let $X$ and $Y$ be two sets. Let $\rho=d(X, Y)$ (Hausdorff distance). Let $B$ denote the disk structuring element with radius 1 . Then the second Hausdorff interpolates, $\left\{Z_{\alpha}: \alpha \in[0,1]\right\}$ is defined as

$$
Z_{\alpha}=\delta_{\alpha \rho B}(X) \bigcap \delta_{(1-\alpha) \rho B}(Y) \bigcap((1-\alpha) X \oplus(\alpha) Y)
$$

The second method above makes up the Second Hausdorff Interpolates as defined in definition 2. The corresponding second Hausdorff interpolates for figure 3 are shown in figure 4. Second Hausdorff interpolates also satisfy (15).

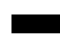

(a)

(e)

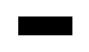

(b)

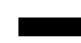

(f)

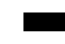

(c)

(d)

$\square$

(h)
Fig. 4. Second Hausdorff Interpolates. (a) Source Image. (b) - (g) Second Hausdorff Interpolate. (h) Target Image. Note that the target image is a simple translation of the object in source image.

\section{INTERPOLATIONS THROUGH MEDIAN}

In this section we look at an alternate method to construct the interpolates. In this section we combine several ideas/assumptions from [23], [30], [10], [2], giving intuitive justifications to each of the assumptions and by providing rigorous statements in support of them. To start with we propose a series of simplifications which reduces and simplifies the problem of finding image interpolates, and then propose a solution to this simplified version of the interpolation problem. We also analyze various properties and explain their significance. We assume that $\left\{Z_{\alpha}\right\}$ indicates the interpolates, with $\alpha$ ranging from 0 to 1 . The proofs of the propositions are given in the appendix for ease of reading.

\section{A. Series Of Simplifications}

Here, we reduce the problem of finding image interpolates to a simpler problem, which would be easier to solve. Recall that binary images can be described as sets in $\mathbb{P}(E)$. In this section, we assume that the problem is to find the interpolates between sets $X$ and $Y$, which are in fact binary images on domain $E$.

1) $X \cap Y \neq \emptyset$ : Consider the case when a set $X$ is deformed by a translation in space to $X_{h}=X+\{h\}$. The ideal interpolates must be of that of a translation, i.e $Z_{\alpha}=X+\{\alpha h\}$. Morphological operators are not equipped to handle such transformations. Indeed, the dilation and erosion operators are extended assuming translation to invariance. Hence, if the operators assume translation invariance, they cannot simulate translation. Hence it makes sense to negate the translation before calculating the interpolates and then adjust the solution accordingly. In fact we negate all affine transformations.

Proposition 1. Let $\mathcal{T}$ denote an affine transformation. Let $X$ and $Y$ be two sets. If $\mathcal{T}(X) \cap Y=\emptyset$ for all $\mathcal{T}$ then either $X$ is empty or $Y$ is empty.

The above proposition is easy to see. Proposition 1 and negating the affine transformations allows the assumption

$$
X \cap Y \neq \emptyset
$$

to hold true. The 'ideal' affine transformation is obtained by

$$
\mathcal{T}^{*}=\arg \max \operatorname{Area}(\mathcal{T}(X) \cap Y)
$$

the affine transformation which maximizes the area of intersection between $X$ and $Y$. This problem is computationally hard, but there exists several approximations [10]. We assume that any affine transformation has 3 parts - translation, rotation and scaling, that is

$$
\mathcal{T}=T_{h} R_{\theta} S_{\lambda}
$$

We can accordingly define,

$$
\mathcal{T}^{-(1-\alpha)}=T_{-(1-\alpha) h} R_{-(1-\alpha) \theta} S_{-(1-\alpha) \lambda}
$$

How to reconstruct the Interpolates? Let interpolates between $\mathcal{T}(X)$ and $(Y)$ be $\left\{Z_{\alpha}^{\prime}\right\}$. Then the interpolates between $X$ and $Y$ is obtained by

$$
Z_{\alpha}=\mathcal{T}^{-(1-\alpha)}\left(Z_{\alpha}^{\prime}\right)
$$

To check this, observe that $Z_{0}=\mathcal{T}^{-(1)}\left(Z_{0}^{\prime}\right)=\mathcal{T}^{-(1)} \mathcal{T}(X)=$ $X$. And similarly we have $Z_{1}=Y$. 
2) $X \subseteq Y:$ Intuitively, an interpolation method between two sets $X$ and $Y$ must gradually remove features from $X$ and gradually incorporate features from $Y$. The features in $X \cap Y$ must remain unchanged. Taking this heuristic into account, to calculate the interpolates between $X$ and $Y$, one can calculate the interpolates between $X$ and $X \cap Y,\left\{U_{\alpha}\right\}$, and interpolates between $Y$ and $X \cap Y,\left\{W_{\alpha}\right\}$. Then the interpolates between $X$ and $Y$ are given by, $\left\{Z_{\alpha}\right\}$, where,

$$
Z_{\alpha}=U_{1-\alpha} \cup W_{\alpha}
$$

Thus we need only develop a method to calculate the interpolates between $X$ and $Y$ under the assumption $X \subseteq Y$, and any such method extends to the general case using (22).

To assess the reasonability of the above simplification, consider the case when the interpolates $U_{\alpha}$ and $W_{\alpha}$ are obtained using definition 2. The following relations then hold true.

$$
\begin{array}{rl}
d\left(X, U_{\alpha}\right)=(1-\alpha) \rho_{1} & d\left(X \cap Y, U_{\alpha}\right)=(\alpha) \rho_{1} \\
d\left(Y, W_{\alpha}\right)=(1-\alpha) \rho_{2} & d\left(X \cap Y, W_{\alpha}\right)=(\alpha) \rho_{2} \\
U_{\alpha} \subset X & W_{\alpha} \subset Y
\end{array}
$$

Here we assumed that $d(X, X \cap Y)=\rho_{1}$ and $d(Y, X \cap Y)=$ $\rho_{2}$. Then the proposition 2 holds true.

Proposition 2. Let $\left\{U_{\alpha}\right\}$ and $\left\{W_{\alpha}\right\}$ be as described above and without loss of generality assume that $\rho_{1} \leq \rho_{2}$. Also assume that $d(X, Y)=\rho_{2}$. Let the interpolates be defined by (22). Then we have

$$
d\left(X, Z_{0.5}\right)=d\left(Y, Z_{0.5}\right)
$$

Note that the assumption $d(X, Y)=\rho_{2}$ needs to be justified. Firstly, note that $d(X, Y) \leq \rho_{2}$ since we have that $d(X \cap Y, Y)=\rho_{2}$. The assumption implies that there exists no point in $X \backslash Y$ closer to $Y$ than in $X \cap Y$ and vice versa. This intuitively follows from the previous simplification which maximizes the area $(X \cap Y)$. Since otherwise, one can find an affine transformation which would increase the value of area $(X \cap Y)$.

Proposition 2 provides a justification for this construction in that, under some conditions, the 'middle' element constructed is equidistant from both $X$ and $Y$. Proposition 3 states an interesting property of the construction above.

Proposition 3. Let $\left\{U_{\alpha}\right\}$ and $\left\{W_{\alpha}\right\}$ be as described above. Let the interpolates be defined by (22). Then we have

$$
d\left(X \cap Y, Z_{0.5}\right)=d\left(X \cup Y, Z_{0.5}\right)
$$

Remark: A Few methods in the existing literature avoid the affine transformation by considering a mask $R$ (usually taken as a convex hull) such that $X, Y \subset R$ [15], [12]. Accordingly, they construct the median $U_{0.5}$ between $X$ and $R$, and $w_{0.5}$ between $Y$ and $R$. The final median is then defined to be

$$
Z_{0.5}=U_{0.5} \cap W_{0.5}
$$

The main difference being - intersections instead of unions. Assuming that medians is self-dual, this is equivalent to the method described above. That is - in the case of $X \cap Y=\emptyset$, consider the sets $X^{c}$ and $Y^{c}$. Then we have that $X^{c} \cap Y^{c} \neq \emptyset$. Construct the median between $X^{c} \cap Y^{c} \rightarrow X^{c}$ and $X^{c} \cap$ $Y^{c} \rightarrow Y^{c}$ and proceed accordingly. If the median constructed is self dual, then we have that median obtained is the same as $X \rightarrow X \cup Y$. This is the same as taking the mask, $R$, to be $X \cup Y$. We reiterate that this equivalence holds only when the median construction is self dual.

3) Construct only medians: Any $\alpha \in[0,1]$ can be written in binary code. Thus, if one has a method to generate only the interpolate $Z_{0.5}$, one can generate the interpolate for any $\alpha$. For example, let $\alpha=5 / 8$. The following steps would generate $Z_{\alpha}$.

(i) Generate interpolate between $Z_{0}$ and $Z_{1}$, we get $Z_{0.5}$.

(ii) Generate interpolate between $Z_{0.5}$ and $Z_{1}$, we get $Z_{0.75}$.

(iii) Generate interpolate between $Z_{0.5}$ and $Z_{0.75}$, we get $Z_{0.625}=Z_{\alpha}$.

The interpolate $Z_{0.5}$ is referred to as the median interpolate. Thus, if one has the method to generate the median then one can generate the interpolate for any $\alpha$. However, note that any such method to produce the median must satisfy the consistency property 1 . For instance, the median between $Z_{0.25}$ and $Z_{0.75}$ must equal the median between $Z_{0}$ and $Z_{1}$.

Property 1 (Consistency of Median). Let $Z_{\alpha_{1}}$ and $Z_{\alpha_{2}}$ be two interpolates. Then the median between $Z_{\alpha_{1}}$ and $Z_{\alpha_{2}}$ must be $Z_{\left(\alpha_{1}+\alpha_{2}\right) / 2}$

Proposition 4. Suppose $X \subseteq X^{\prime} \subseteq Y^{\prime} \subseteq Y$ and $m(X, Y)$ denotes the median between $X$ and $Y$ then

$$
\begin{aligned}
m(X, Y)= & m\left(X^{\prime}, Y^{\prime}\right) \Rightarrow \\
& m(X, Y)=m\left(m\left(X, X^{\prime}\right), m\left(Y, Y^{\prime}\right)\right) \\
& m\left(Z_{0}, Z_{1}\right)=m\left(Z_{0.25}, Z_{0.75}\right)
\end{aligned}
$$

if (26) and (27) holds true then the property 1 holds as well.

Note that consistency of the medians is a desired property. However, the heuristic is still valid and can be followed to reduce the computational costs. Remark: One can also selectively generate the medians between the frames with highest error rates instead [11]. This can further reduce the computation costs.

\section{B. Median}

In [30] a procedure to construct the median is proposed, as per definition 3 below. This is referred henceforth as Serra's median.

Definition 3 (Serra's Median). Given two sets $X \subseteq Y$, the median $m(X, Y)$ is defined as

$$
m(X, Y)=\bigcup_{\lambda \geq 0}(\{X \oplus \lambda B\} \cap\{Y \ominus \lambda B\})
$$

Serra's median defined above can also be characterized in terms of influence zones as shown in proposition 5. 
Proposition 5. Let $X \subseteq Y$ be two sets and $B$ be the disk structuring element. Let Serra's median be defined as in (28). Then we have that

$$
m(X, Y)=I Z\left(X \mid Y^{c}\right)
$$

Another property we expect from a median is duality. That is, we expect that $m(X, Y)=\left(m\left(Y^{c}, X^{c}\right)\right)^{c}$ to hold. Serra's median does not satisfy this property, but one can show that they are 'close enough'. The dual Serra's median is defined as

$$
M(X, Y)=\left(m\left(Y^{c}, X^{c}\right)\right)^{c}
$$

Proposition 6. Let $X \subseteq Y$ be two sets. Let $m(X, Y)$ be Serra's median as defined in (28). Let $M(X, Y)$ be the dual Serra's median as defined in (30). Then we have

$$
M(X, Y)=\bigcap_{\lambda>0}\{(Y \ominus \lambda B) \cup(X \oplus \lambda B)\}
$$

and

$$
M(X, Y) \backslash m(X, Y)=\{x \mid \bar{d}(X, x)=\bar{d}(Y, x)\}
$$

Thus although, duality does not hold, we have that Serra's median and the dual Serra's median only differ in the boundary.

Recall that we have stated earlier that it is favorable for median to satisfy the consistency property 1 . Although, Serra's median defined above satisfy this property for simple sets, it is still an open question as to whether it is true for all sets $X \subseteq Y$. We discuss further on this in section VII.

Another problem with Serra's median arises in the case of non-convex shapes. Consider the sets $X$ and $Y$ as in figure 5 (a) and (b). One expects the median to be close to figure 5(e) while Serra's median calculation results in 5(c).

\section{Meyer's Method}

We now describe yet another method to calculate the interpolates between two images, thanks to F. Meyer, as described in [23]. Given two sets $X$ and $Y$, we are required to find a median. As mentioned earlier, we assume that $X \subseteq Y$. Let $x$ be a point in the set $Y \backslash X$. Recall $\bar{d}(X, x)=\inf \{\lambda \mid x \in X \oplus \lambda B\}$. For this section we denote $\bar{d}(X, x)=d_{\delta}(X, x)$ to be consistent with the notation in [23]. Let $d_{\epsilon}(x, Y)=\inf \{\lambda \mid x \notin Y \ominus \lambda B\}$. Define

$$
f(x)= \begin{cases}1 & x \in X \\ \frac{d_{\epsilon}(x, Y)}{d_{\epsilon}(x, Y)+d_{\delta}(X, x)} & x \in Y \backslash X \\ 0 & x \in Y^{c}\end{cases}
$$

We thus have a greyscale image with values in $[0,1]$. To calculate the interpolate, we take

$$
Z_{\alpha}=\mathbb{T}_{\alpha}(f)
$$

where, $\mathbb{T}_{\alpha}($.$) is a threshold operator and f$ is the greyscale image obtained by (33). This formulation gives us an easy and efficient way to calculate the interpolates. An example is shown in figure 5(d). Note that, (33) suffers from the same problem as that of Serra's median.

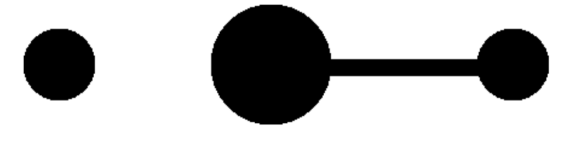

(a)

(c)
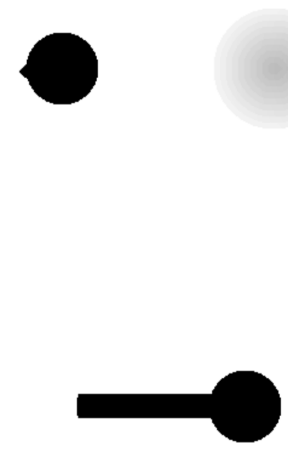

(e) (b)

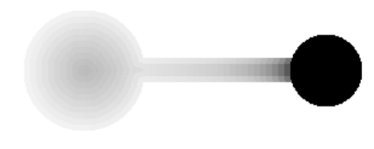

(d)
Fig. 5. (a) Set $X$. (b) Set $Y$ (c) Serra's median calculated according to (28). (d) The grey scale image obtained by Meyer's method. (e) Median calculated using (35).

An alternative simple solution was proposed in [4] to get the interpolates in these kind of situations. Assume that $X \subseteq Y$. Let $\bar{d}(X, Y)=\rho$. For all $x \in Y \backslash X$. Define

$$
f(x)= \begin{cases}1 & x \in X \\ 1-\frac{d_{\delta}(X, x)}{\rho} & x \in Y \backslash X \\ 0 & x \in Y^{c}\end{cases}
$$

Thresholding the greyscale image in (35) gives the interpolates. This method works better in cases as in figure 5. The median image in figure 5(e) is obtained using this method. Meyer's method is related to the one in definition 3 as shown in following proposition.

Proposition 7. Let $X \subseteq Y$ be two sets. Serra's median calculated by (28), is the same as one obtained by (34), taking $\alpha=0.5$.

Note that although Meyer's median and Serra's median are the same, in general this need not be true for all interpolates. The main advantage of Meyer's method is that one can calculate all the interpolates simultaneously and this saves a lot of computation.

Note that the methods described above in (33) and (35) are proposed in a generalized framework as

$$
f(x)= \begin{cases}1 & x \in X \\ 1-\frac{d_{\delta}(X, x)}{k\left(d_{\epsilon}(x, Y)+d_{\delta}(X, x)\right)+(1-k) \rho} & x \in Y \backslash X \\ 0 & x \in Y^{c}\end{cases}
$$


referred to as universal morphological interpolator. There are several other advantages of universal morphological interpolator as well [15], [13].

\section{GReysCAle IMAGeS}

Recall that the operators of binary images are extended to greyscale images using subgraphs. An important point to note is that not all the three dimensions can be treated the same. Two dimensions belong to the spatial domain, and the third gives the value domain. This impacts the way structuring elements would be handled. This results in two kinds of structuring elements - flat and non-flat as discussed in II-B. In this section we first look at how the interpolation methods discussed above extend to greyscale images. We then analyze the distinction between flat and non-flat structuring elements.

Firstly, note that simply replacing the binary dilation/erosion with greyscale dilation/erosion will enable to extend the interpolations in definitions 1 and 2 to greyscale images as well. Since greyscale images are just sets (subgraphs) in a different space, all the properties generalize accordingly.

Calculating Serra's median as per definition 3, one needs to verify the validity of series of simplifications in section IV-A. The assumption $1, X \cap Y \neq \emptyset$ trivially holds for greyscale images since we consider the subgraphs. This is because of having a value domain. Assumption 2, $X \subset Y$ also holds for greyscale images, since we still are considering sets, although in higher dimensions. Assumption 3, that medians are enough, also would apply greyscale images. Thus, if $f_{1}$ and $f_{2}$ are two 1 -d greyscale images, we assume that $f_{1} \geq f_{2}$ and the problem of interpolation drops to finding the median element.

Meyer's median can also be extended to greyscale images using the the idea of $3 \mathrm{~d}$ interpolators and is discussed in [14]. In this section, we only analyze the medians constructed hierarchically, using Serra's median.

\section{A. Flat vs Non-Flat structuring elements}

We say that we get 'thin' interpolates between two sets $X \subseteq Y$ if the $S K I Z\left(X, Y^{c}\right)$ has empty interior. Otherwise they are called 'thick' interpolates. It is easy to see that in binary images we are assured of getting thin interpolates using influence zones. This does not extend to greyscale images. Recall that

$$
\operatorname{SKIZ}\left(X, Y^{c}\right)=\left\{x \mid \bar{d}(X, x)=\bar{d}\left(Y^{c}, x\right)\right\}
$$

In case of binary images, for any $X$ and $x$ we have that $\bar{d}(X, x)<\infty$. Intuitively, this is the reason why one gets thin interpolates. However, if we consider a greyscale image and a flat structuring element, then it is possible that $\bar{d}(X, x)=\infty$. Thus, thin interpolates are not assured when considering greyscale images and using flat structuring elements. However, a transformation on the greyscale value domain would allow us to obtain thin interpolates.

Proposition 8. Let $f_{1}(x) \geq f_{2}(x)$ for all $x$, be two greyscale images defined on finite domain $E \subset \mathbb{R}^{2}$. If, $\inf f_{1} \leq \sup f_{2}$, then $S K I Z\left(f_{2}, f_{1}^{c}\right)$ has the empty interior, when influence zones are calculated using flat structuring elements.
The proof of proposition 8 follows from noting that in case we have inf $f_{1} \leq \sup f_{2}$, then for all $x$ either $\bar{d}\left(f_{2}, x\right)<\infty$ or $\bar{d}\left(f_{1}^{c}, x\right)<\infty$. This implies that case when $\bar{d}\left(f_{2}, x\right)=$ $\bar{d}\left(f_{1}^{c}, x\right)=\infty$ does not happen, and hence the proposition follows [38], [21].

The above proposition implies that under some regularity conditions, thick boundaries do not appear even with flat structuring elements. It is not tough to see that, there exists a transformation on the value domain, under which one can guarantee $\inf f_{1} \leq \sup f_{2}$. A simple transformation is to take

$$
f(x) \rightarrow \frac{f(x)-\min (f)}{\max (f)-\min (f)}
$$

Note that this transformation results in the changes to brightness and contrast in an image. We assume that whenever the method uses flat structuring elements, this transformation is suitably applied.

In summary, the procedure to calculate the median according to definition 3 is

1) Let $f_{1}$ and $f_{2}$ be two greyscale images.

2) Calculate $\hat{f}=\min \left(f_{1}, f_{2}\right)$.

3) Calculate the medians between $f_{1}$ and $\hat{f},\left\{x_{\alpha}\right\}$ and $f_{2}$ and $\hat{f}\left\{y_{\alpha}\right\}$. If using flat structuring elements, appropriately scale $f_{1}$ and $f_{2}$.

4) The median between $f_{1}$ and $f_{2}$ is then given by $\sup \left\{x_{1-\alpha}, y_{\alpha}\right\}$.

5) Repeat the above steps iteratively to get the interpolates.

Which to choose- Flat or Non-Flat? Usually, Non-flat structuring elements are preferred since (1) they do not result in thick interpolates and (2) Flat structuring elements does not necessarily converge to the source/target images. However, the proposition 8 states that even flat structuring elements can be used and still avoid thick interpolates under some conditions. In practice, it has been observed that non-flat structuring elements gives smoother interpolates as illustrated in the following example.

Example - Simulated shoreline interpolation: As an example to illustrate the difference between flat and non-flat structuring elements, we simulate shorelines at two distinct times and calculate the interpolates. Evolving shorelines has also been studied in [31], using medians to extrapolate the shorelines. We simulate the shorelines by considering two 1dimensional functions

$$
\begin{aligned}
& f_{1}=N(2,0.5)+N(4,1)+N(7,1.5) \\
& f_{2}=N(1,1)+N(3,0.5)+N(8,1.5)
\end{aligned}
$$

where,

$$
N(\mu, \sigma)=\exp \left\{\frac{1}{2}\left(\frac{x-\mu}{\sigma}\right)^{2}\right\}
$$

The functions $f_{1}$ and $f_{2}$ are shown in figures 6 (a) and (b). The median obtained by flat/non-flat structuring elements is plotted in figures 6 (c) and (d) respectively. It can be seen that the median calculated using non-flat structuring element takes into account the shape of $f_{1}$ better than the median using flat structuring element, between 4 and 8 on the $\mathrm{x}$-axis. This is because, in that patch the flat structuring element cannot go below the minimum. This in effect shows that proposition 8 


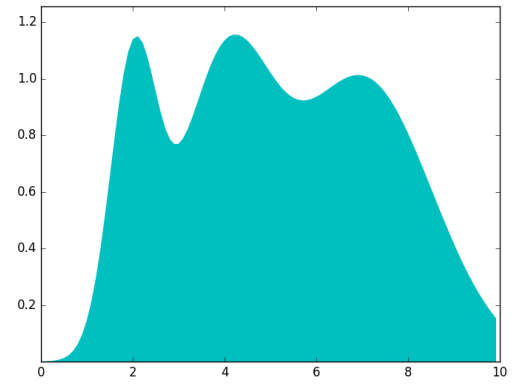

(a)

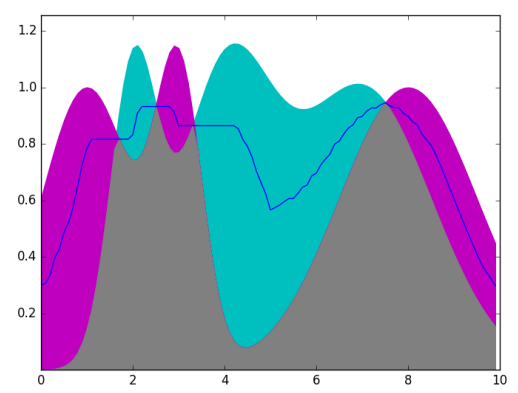

(d)

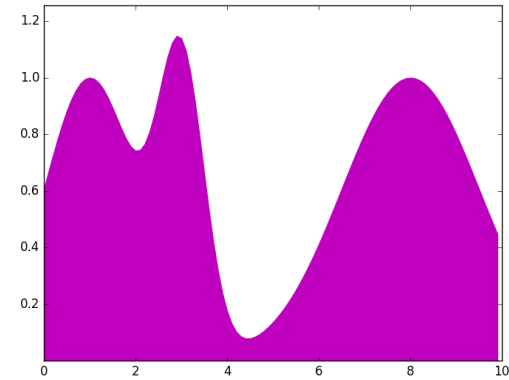

(b)

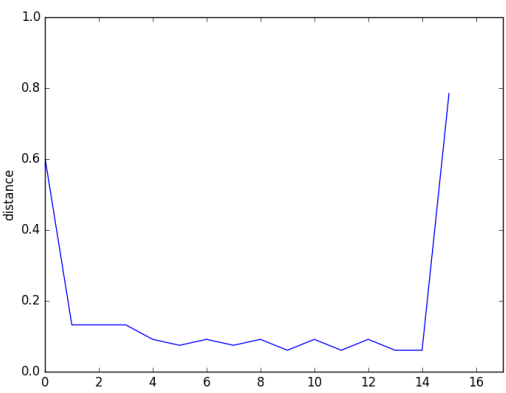

(e)

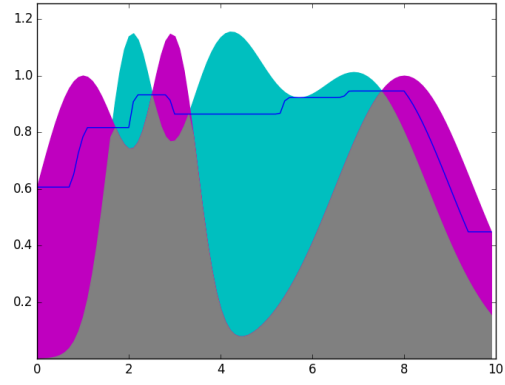

(c)

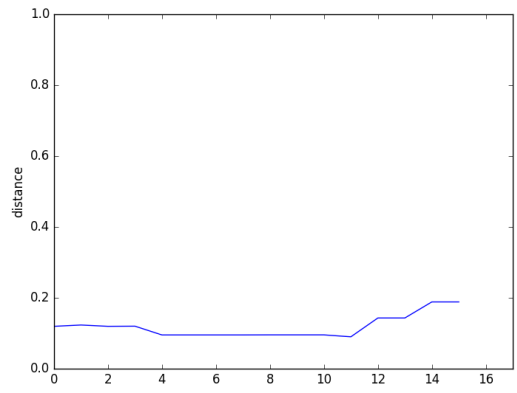

(f)

Fig. 6. (a) Shoreline at time $t=0$ denoted by the cyan region. (b) Shoreline at time $t=1$ denoted by the magenta region. The grey region indicates the part which did not change between times $t=0$ and $t=1$. (c) Median (boundary) obtained by flat structuring element indicated by the blue line . (d) Median (boundary) obtained by non-flat structuring element indicated by the blue line . (e) Plot of the distances as in (40) between successive interpolates obtained by flat structuring element. (f) Plot of the distances as in (40) between successive interpolates obtained by non-flat structuring element.

is not enough to justify the use of flat structuring elements instead of non-flat structuring elements.

$$
L^{\infty}\left(f_{1}, f_{2}\right)=\sup _{x}\left|f_{1}(x)-f_{2}(x)\right|
$$

To analyze the difference further, hierarchical medians were generated to the level 4 (which gives 16 interpolates in total) for both flat and non-flat structuring elements. Then distance measured by $L^{\infty}$ (40) is calculated between successive interpolates and plotted in figures 6 (e) and (f). From this, one can deduce that non-flat structuring elements gives smoother interpolates compared to the flat structuring elements especially at the ends. This is because, even though proposition 8 holds true on the whole domain, it fails in the interior (for instance between 4 and 6 in figure 6 ). So, in order to use flat structuring elements to generate the whole set of interpolates, one has to divide the domain into finer parts and proceed accordingly.

\section{EXTENSIONS AND FUTURE WORK}

As stated earlier, another important aim of this paper is to provide a platform for various extensions and future work. In this section, we pose several questions, discuss their importance and possible questions to be answered.

\section{A. Graph based Interpolation}

A graph $\mathcal{G}=(V, E)$ is a tuple of a vertex set $V$ and an edge set $E \subseteq V \times V$. An image can be represented as a graph, taking the set of pixels as the vertex set and edges between two adjacent pixels. Recently, MM operators are generalized to graphs [6], [8], [25]. So, a natural question arises - Can we extend the morphological interpolates to graphs as well? We briefly review the question here.

The basic MM operators, dilation and erosion, can be extended to the graphs using the lattice definitions - dilation defined as the operator which preserves the supremum and erosion being the operator which preserves the infimum. There are in fact two different kinds of dilations/erosions one can define $-\delta^{\bullet}, \delta^{\times}, \epsilon^{\bullet}, \epsilon^{\times}$. (Complete details can be found in [6]). The operators $\delta^{\bullet}, \epsilon^{\bullet}$ map the set of edges to a set of vertices while the operators $\delta^{\times}, \epsilon^{\times}$map a set of vertices to a set of edges.

Defining,

$$
\begin{aligned}
\Delta(X) & =\delta^{\bullet}\left(\delta^{\times}(X)\right) \\
\mathcal{E}(X) & =\epsilon^{\bullet}\left(\epsilon^{\times}(X)\right)
\end{aligned}
$$

allows us to define the dilation and erosion operators on the set of vertices to vertices. All the above methods to obtain binary interpolates can be directly extended to graphs using these operators instead.

There is also another possibility of extending the operators using only $\delta^{\times}$or $\delta^{\bullet}$. Each of these operators can be thought of as half-dilations. To represent these, one has to use a different representation of the image using cubical complexes as established in [16]. 
For greyscale images, one can think of them as binary images in higher dimension as in (5), construct a graph and then proceed as above. However, an alternate solution would be to extend the graph based MM operators to edge weighted graphs and accordingly define the interpolates. The main advantage of extending the framework to graphs is several kinds of data can be represented in terms of edgeweighted graphs. Extending the techniques (non graph based) discussed to RGB scale is non trivial since there is no natural order. This presents a problem with extending the techniques to other domains (in particular for hyperspectral images). On the other hand, it is widely followed practice to convert data into edge weighted graphs and there exists several methods [35]. Thus, extending the techniques of morphological interpolation to graphs would prove useful.

Apart from above, this would allow us to use morphological interpolation techniques for the image upscaling problem using label propagation [20]. The topic of analyzing the graph based interpolations on graphs is a topic of further research.

\section{B. Problem of median being consistent}

Recall that in property 1 we stated that any median must be consistent, and in proposition 4 we have given an equivalent condition for the property to hold. It can be easily seen that the median obtained by using (35) follows this property. However, it is still an open question whether medians proposed in definition 3 satisfies this property or not. Apart from this, other equivalent conditions to the consistency property $1 \mathrm{might}$ provide better insights into the interpolation problem.

\section{Loss function for judging interpolates}

An important task is to have an ability of judging interpolates obtained via a loss function. This would allow us to compare different methods of interpolation and maybe obtain new interpolation methods as well. The basic criteria for such a function is

- It should have higher values for non-smooth interpolates compared to smoother interpolates

- Should be independent of the number of interpolates obtained.

As a starting point, one can consider the following metric. Let $d\left(I_{1}, I_{2}\right)$ be any metric to measure the distance between two images. For instance $L^{\infty}$ measure in(40). Let $\mathcal{Z}=\left\{I_{0}=\right.$ $\left.Z_{0}, Z_{1}, Z_{2}, \cdots, Z_{n}=I_{1}\right\}$ be a set of interpolates. Define,

$$
D(\mathcal{Z})=\sup _{0<i<n-1} d\left(Z_{i}, Z_{i+1}\right)
$$

This satisfies the condition to penalize non-smooth interpolates. However, increasing the number of interpolates can reduce this metric. Finding a right metric to judge interpolates is also a subject of future research.

Another advantage of having a loss function is that, one can pose the problem of interpolation as an optimization problem which would allow us to solve the interpolation problem by making use of the vast number of techniques in the area of optimization.

\section{CONCLUSION}

In this article we reviewed some methods to get a solution to the problem of image interpolation. Constraining the data domain to -science and remote sensing allowed to justify the use of the MM operators (which heuristically simulate geophysical processes). We have reviewed several methods to construct medians and provided the links between existing methods. We have discussed the validity of various assumptions (section IV-A). The argument of flat vs non-flat structuring elements was discussed in section VI. Also, discussed are the problems of future research extending the techniques discussed.

\section{ACKNOWLEDGMents}

The authors would like to thank the associate editor Jiang, Xudong for his consideration, and the anonymous reviewers for their valuable comments and providing references. The authors would also like to thank Prof. Jean Serra for providing us with references to his work. The authors AC and SD would like to thank Indian Statistical Institute for the fellowship provided to pursue the above research.

\section{APPENDIX}

\section{PROOF FOR PROPOSITION 2}

Proof. Firstly, note that

$$
\begin{aligned}
\bar{d}\left(X, Z_{\alpha}\right)=\bar{d}\left(X, W_{\alpha}\right) & \leq \bar{d}\left(X \cap Y, W_{\alpha}\right)=\alpha \rho_{2} \\
\bar{d}\left(Z_{\alpha}, X\right) \leq \bar{d}\left(U_{1-\alpha}, X\right) & =\alpha \rho_{1}
\end{aligned}
$$

Hence we have,

$$
d\left(X, Z_{\alpha}\right) \leq \sup \left\{\alpha \rho_{1}, \alpha \rho_{2}\right\}
$$

Taking $\alpha=0.5$, we have that

$$
d\left(X, Z_{0.5}\right) \leq 0.5 \rho_{2}
$$

Similarly, we also have that

$$
d\left(Y, Z_{0.5}\right) \leq 0.5 \rho_{2}
$$

From triangle inequality,

$$
\begin{aligned}
\rho_{2} & =d(X, Y) \\
& \leq d\left(X, Z_{0.5}\right)+d\left(Y, Z_{0.5}\right) \\
& \leq 0.5 \rho_{2}+0.5 \rho_{2}=\rho_{2}
\end{aligned}
$$

Hence we can deduce that

$$
d\left(X, Z_{0.5}\right)=d\left(Y, Z_{0.5}\right)
$$




\section{PROOF FOR PROPOSITION 3}

Proof. Assume, without loss of generality that $\rho_{1} \leq \rho_{2}$. Then we have that

$$
\begin{aligned}
d\left(X \cap Y, Z_{0.5}\right) & =\sup \left\{d\left(X \cap Y, W_{0.5}\right), d\left(X \cap Y, U_{0.5}\right)\right\} \\
& =\sup \left\{0.5 \rho_{1}, 0.5 \rho_{2}\right\}=0.5 \rho_{2}
\end{aligned}
$$

Also, we have that

$$
d(X \cap Y, X \cup Y)=\sup \{d(X \cap Y, X), d(X \cap Y, Y)\}=\rho_{2}
$$

From (42) we have

$$
\begin{aligned}
d\left(X, Z_{\alpha}\right) & \leq \sup \left\{\alpha \rho_{2}, \alpha \rho_{1}\right\} \\
d\left(Y, Z_{\alpha}\right) & \leq \sup \left\{\alpha \rho_{2}, \alpha \rho_{1}\right\}
\end{aligned}
$$

Taking $\alpha=0.5$ we have

$$
\begin{aligned}
d\left(Z_{0.5}, X \cup Y\right) & =\sup \left\{d\left(Z_{0.5}, X\right), d\left(Z_{0.5}, Y\right)\right\} \\
& \leq 0.5 \rho_{2}
\end{aligned}
$$

Using triangle inequality,

$$
\begin{aligned}
\rho_{2} & =d(X \cap Y, X \cup Y) \\
& \leq d\left(X \cap Y, Z_{0.5}\right)+d\left(Z_{0.5}, X \cup Y\right) \\
& \leq 0.5 \rho_{2}+0.5 \rho_{2}=\rho_{2}
\end{aligned}
$$

From this we can deduce that

$$
d\left(X \cap Y, Z_{0.5}\right)=d\left(X \cup Y, Z_{0.5}\right)=0.5 \rho_{2}
$$

\section{PROOF OF PROPOSITION 4}

Proof. Here we only provide the intuitive idea of why the proposition is correct. Let $Z_{0}=X \subset Y=Z_{1}$. Indicate the median, $m\left(Z_{\alpha_{1}}, Z_{\alpha_{2}}\right)$ by $Z_{\left(\alpha_{1}+\alpha_{2}\right) / 2}$. Thus $Z_{0.5}=$ $m\left(Z_{0}, Z_{1}\right)$. Assume that,

$$
Z_{0.5}=m\left(Z_{0.25}, Z_{0.75}\right)=m\left(Z_{0}, Z_{1}\right)=m\left(Z_{0.5}, Z_{0.5}\right)
$$

Using (26) we get,

$$
Z_{0.5}=m\left(Z_{0.125}, Z_{0.875}\right)=m\left(Z_{0}, Z_{1}\right)=m\left(Z_{0.375}, Z_{0.625}\right)
$$

One can continue this to show that, for all $\epsilon<0.5$

$$
Z_{0.5}=m\left(Z_{0.5-\epsilon}, Z_{0.5+\epsilon}\right)
$$

This can be generalized to any $\alpha$, that is

$$
Z_{\alpha}=m\left(Z_{\alpha-\epsilon}, Z_{\alpha+\epsilon}\right)
$$

by observing that sets $X$ and $Y$ are arbitrary. So, the first time we encounter $\alpha$, we can name the sets $Z_{0}$ and $Z_{1}$ and proceed accordingly.

\section{PROOF OF PROPOSITION 5}

Proof. We have

$$
\begin{aligned}
I Z\left(X \mid Y^{c}\right) & =\bigcup_{\lambda \geq 0}\left\{(X \oplus \lambda B) \cap\left(Y^{c} \oplus \lambda B\right)^{c}\right\} \\
& =\bigcup_{\lambda \geq 0}\{(X \oplus \lambda B) \cap(Y \ominus \lambda B)\} \\
& =m(X, Y)
\end{aligned}
$$

The first equality follows from (12). The second equality comes from the duality between dilation and erosion operators [24].

\section{PROOF OF PROPOSITION 6}

Proof. Equation (31) can be obtained by set transformations. To show (32) - Since, we have that, from proposition 5.

$$
m(X, Y)=I Z\left(X \mid Y^{c}\right)=\left\{x \mid \bar{d}(X, x)<\bar{d}\left(Y^{c}, x\right)\right\}
$$

and

$$
\begin{aligned}
M(X, Y) & =\left(m\left(X^{c}, Y^{c}\right)\right)^{c} \\
& =\left(I Z\left(Y^{c} \mid X\right)\right)^{c}=\left\{x \mid \bar{d}\left(Y^{c}, x\right)<\bar{d}(X, x)\right\}^{c} \\
& =\left\{x \mid \bar{d}(X, x) \leq \bar{d}\left(Y^{c}, x\right)\right\}
\end{aligned}
$$

Thus we have,

$$
M(X, Y) \backslash m(X, Y)=\{x \mid \bar{d}(X, x)=\bar{d}(Y, x)\}
$$

\section{PROOF OF PROPOSITION 7}

Proof. The proof follows from the fact that, at $\alpha=0.5$ we have that the Meyer's median consists of

$$
\left\{x: d_{\delta}(X, x) \leq d_{\epsilon}(x, Y)\right\}
$$

In other words, the Meyer's median is equal to $I Z\left(X \mid Y^{c}\right)$, which from proposition 5 equals the one in (28).

\section{REFERENCES}

[1] J. Angulo and J. Serra, "Mathematical morphology in color spaces applied to the analysis of cartographic images," Proceedings of GEOPRO, vol. 3, pp. 59-66, 2003.

[2] S. Beucher, "Sets, partitions and functions interpolations," Computational Imaging and Vision, vol. 12, pp. 307-314, 1998.

[3] G. Birkhoff, Lattice Theory, ser. American Mathematical Society colloquium publications. American Mathematical Society, 1940, no. v. 25, pt. 2. [Online]. Available: https://books.google.co.in/books?id= ePqVAwAAQBAJ

[4] A. Challa, S. Danda, and B. S. D. Sagar, "Morphological interpolation for temporal changes," in Geoscience and Remote Sensing Symposium (IGARSS), 2016 IEEE International. IEEE, 2016, pp. 3358-3361.

[5] V. Chatzis and I. Pitas, "Interpolation of 3-d binary images based on morphological skeletonization," IEEE transactions on medical imaging, vol. 19, no. 7, pp. 699-710, 2000.

[6] J. Cousty, L. Najman, F. Dias, and J. Serra, "Morphological filtering on graphs," Computer Vision and Image Understanding, vol. 117, no. 4, pp. 370-385, 2013.

[7] G. Grätzer, Lattice Theory: Foundation, ser. SpringerLink : Bücher Springer Basel, 2011. [Online]. Available: https://books.google.co.in/ books?id=6XJX5-zCoIQC

[8] H. Heumans, P. Nacken, A. Toet, and L. Vincent, "Graph morphology," Journal of visual communication and image representation, vol. 3, no. 1 , pp. 24-38, 1992.

[9] M. Iwanowski and J. Serra, "Morphological interpolation and color images," in Image Analysis and Processing, 1999. Proceedings. International Conference on. IEEE, 1999, pp. 50-55.

[10] —_ "The morphological-affine object deformation," in Mathematical Morphology and its Applications to Image and Signal Processing. Springer, 2000, pp. 81-90.

[11] M. Iwanowski, "Image morphing based on morphological interpolation combined with linear filtering," International Journal of WSCG, vol. 1 , pp. 233-239, 2002.

[12] - "Morphological binary interpolation with convex mask," in Proceedings International Conference on Computer Vision and Graphics, Zakopane, Poland, 2002.

[13] — , "Universal morphological interpolator," in Image Processing, 2005. ICIP 2005. IEEE International Conference on, vol. 2. IEEE, 2005, pp. II-978.

[14] — "Graytone image metamorphosis using 3d interpolation function." in VISAPP (2), 2009, pp. 5-9. 
[15] _ - "Image methamorphosis based on universal morphological interpolator," Przeglad Elektrotechniczny, vol. 87, no. 9a, pp. 234-237, 2011.

[16] E. Khalimsky, R. Kopperman, and P. R. Meyer, "Computer graphics and connected topologies on finite ordered sets," Topology and its Applications, vol. 36, no. 1, pp. 1-17, 1990.

[17] A. Ledda, H. Q. Luong, W. Philips, V. De Witte, and E. E. Kerre, "Binary image interpolation based on mathematical morphology," in Signal Processing Conference, 2008 16th European. IEEE, 2008, pp. $1-5$.

[18] S.-Y. Lee, K.-Y. Chwa, J. K. Hahn, and S. Y. Shin, "Image morphing using deformation techniques," Journal of Visualization and Computer Animation, vol. 7, no. 1, pp. 3-23, 1996.

[19] X. Li and M. T. Orchard, "New edge-directed interpolation," IEEE transactions on image processing, vol. 10, no. 10, pp. 1521-1527, 2001.

[20] X. Liu, D. Zhao, J. Zhou, W. Gao, and H. Sun, "Image interpolation via graph-based bayesian label propagation," IEEE Transactions on Image Processing, vol. 23, no. 3, pp. 1084-1096, 2014.

[21] L. Loveland, "When midsets are manifolds," Proceedings of the American Mathematical Society, vol. 61, no. 2, pp. 353-360, 1976.

[22] G. Matheron, Random sets and integral geometry, ser. Wiley series in probability and mathematical statistics: Probability and mathematical statistics. Wiley, 1975. [Online]. Available: https: //books.google.co.in/books?id=bgzvAAAAMAAJ

[23] F. Meyer, "A morphological interpolation method for mosaic images," in Mathematical Morphology and its Applications to Image and Signal Processing. Springer, 1996, pp. 337-344.

[24] L. Najman and H. Talbot, Mathematical morphology. John Wiley \& Sons, 2013.

[25] L. Najman and J. Cousty, "A graph-based mathematical morphology reader," Pattern Recognition Letters, vol. 47, pp. 3-17, 2014.

[26] B. S. D. Sagar, "Visualization of spatiotemporal behavior of discrete maps via generation of recursive median elements," Pattern Analysis and Machine Intelligence, IEEE Transactions on, vol. 32, no. 2, pp. 378-384, 2010

[27] B. S. D. Sagar and S. L. Lim, "Morphing of grayscale dems via morphological interpolations," IEEE Journal of Selected Topics in Applied Earth Observations and Remote Sensing, vol. 8, no. 11, pp. 5190-5198, 2015.

[28] P. Salembier and J. Serra, "Flat zones filtering, connected operators, and filters by reconstruction," IEEE Transactions on image processing, vol. 4, no. 8, pp. 1153-1160, 1995.

[29] J. Serra, Image Analysis and Mathematical Morphology. Orlando, FL, USA: Academic Press, Inc., 1983.

[30] _ , "Hausdorff distances and interpolations," Computational Imaging and Vision, vol. 12, pp. 107-114, 1998.

[31] —_, "Shoreline extrapolations," Hornickà prúbram ve vĕdĕ a technice, 2011, available at http://slon.diamo.cz/hpvt/2011/_g+m/MG\%2001.pdf. Last accessed on 2017-03-10. [Online]. Available: http://slon.diamo.cz/ hpvt/2011/_g+m/MG\%2001.pdf

[32] J. Serra and P. Soille, Mathematical morphology and its applications to image processing, ser. Computational imaging and vision. Springer Netherlands, 1994.

[33] L. Vincent, "Morphological grayscale reconstruction in image analysis: Applications and efficient algorithms," IEEE transactions on image processing, vol. 2, no. 2, pp. 176-201, 1993.

[34] D. N. Vizireanu, S. Halunga, and O. Fratu, "A grayscale image interpolation method using new morphological skeleton," in Telecommunications in Modern Satellite, Cable and Broadcasting Service, 2003. TELSIKS 2003. 6th International Conference on, vol. 2. IEEE, 2003, pp. 519521.

[35] U. Von Luxburg, "A tutorial on spectral clustering," Statistics and computing, vol. 17, no. 4, pp. 395-416, 2007.

[36] Z. Wei and K.-K. Ma, "Contrast-guided image interpolation," IEEE Transactions on Image Processing, vol. 22, no. 11, pp. 4271-4285, 2013

[37] R. T. Whitaker, "A level-set approach to image blending," IEEE Transactions on Image Processing, vol. 9, no. 11, pp. 1849-1861, 2000.

[38] J. Wilker, "Equidistant sets and their connectivity properties," Proceedings of the American Mathematical Society, vol. 47, no. 2, pp. 446-452, 1975.

[39] L. Zhu, Y. Yang, S. Haker, and A. Tannenbaum, "An image morphing technique based on optimal mass preserving mapping," IEEE Transactions on Image Processing, vol. 16, no. 6, pp. 1481-1495, 2007.

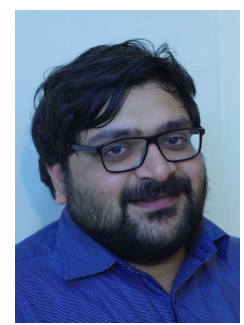

Aditya Challa received the B.Math.(Hons.) degree in Mathematics from the Indian Statistical Institute - Bangalore, and Masters in Complex Systems from University of Warwick, UK - in 2010, and 2012, respectively. From 2012 to 2014, he worked as a Business Analyst at Tata Consultancy Services, Bangalore. In 2014, he joined as a Junior Research Fellow at Indian Statistical Institute - Bangalore, where he is currently a Senior Research Fellow in the Systems Science and Informatics Unit. His current research interests focus on the solutions to the Image Interpolation Problem, and using techniques from Mathematical Morphology in Data Mining.

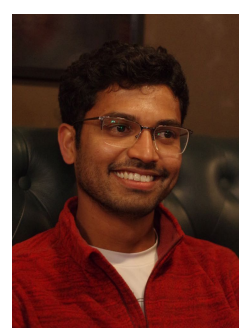

Sravan Danda received the B.Math.(Hons.) degree in Mathematics from the Indian Statistical Institute - Bangalore, and the M.Stat. degree in Mathematical Statistics from the Indian Statistical Institute Kolkata, in 2009, and 2011, respectively. From 2011 to 2013, he worked as a Business Analyst at Genpact - Retail Analytics, Bangalore. In 2013, he joined as a Junior Research Fellow at Indian Statistical Institute - Bangalore, where he is currently a Senior Research Fellow in the Systems Science and Informatics Unit under the joint supervision of B.S.Daya Sagar and Laurent Najman. His current research interests focus on the development of tools for Image filtering and segmentation using combinatorial optimization and Discrete Mathematical Morphology.

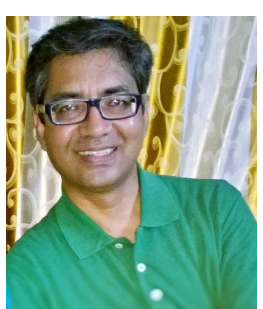

B. S. Daya Sagar (M03-SM03) is a full Professor of the Systems Science and Informatics Unit (SSIU) at the Indian Statistical Institute. Sagar received his $\mathrm{MSc}$ and $\mathrm{PhD}$ degrees in geoengineering and remote sensing from the Faculty of Engineering, Andhra University, Visakhapatnam, India, in 1991 and 1994 respectively. He is also first Head of the SSIU. Earlier, he worked in the College of Engineering, Andhra University, and Centre for Remote Imaging Sensing and Processing (CRISP), The National University of Singapore in various positions during 1992-2001. He served as Associate Professor and Researcher in the Faculty of Engineering and Technology (FET), Multimedia University, Malaysia, during 2001-2007. Since 2017, he has been a Visiting Professor at the University of Trento, Trento, Italy. His research interests include mathematical morphology, GISci, digital image processing, fractals and multifractals, their applications in extraction, analyses, and modelling of geophysical patterns. He has published over 85 papers in journals, and has authored and/or guest edited 11 books and/or special theme issues for journals. He recently authored a book entitled "Mathematical Morphology in Geomorphology and GISci," CRC Press: Boca Raton, 2013, p. 546. He recently co-edited two special issues on "Filtering and Segmentation with Mathematical Morphology" for IEEE Journal of Selected Topics in Signal Processing (v. 6, no. 7, p. 737-886, 2012), and "Applied Earth Observation and Remote Sensing in India" for IEEE Journal of Selected Topics in Applied Earth Observation and Remote Sensing (v. 10, no. 12, p. 5149-5328, 2017). He is an elected Fellow of Royal Geographical Society (1999), Indian Geophysical Union (2011), and was a member of New York Academy of Science during 1995-1996. He received the Dr. Balakrishna Memorial Award from Andhra Pradesh Academy of Sciences in 1995, the Krishnan Gold Medal from Indian Geophysical Union in 2002, and the "Georges Matheron Award-2011 (with Lecturership)" of the International Association for Mathematical Geosciences. He is the Founding Chairman of Bangalore Section IEEE GRSS Chapter. He is on the Editorial Boards of Computers and Geosciences, and Frontiers: Environmental Informatics. 


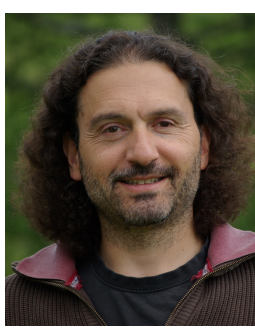

Laurent Najman (SM17) received the Habilitation à Diriger les Recherches in 2006 from University the University of Marne-la-Vallée, a Ph.D. of applied mathematics from Paris-Dauphine University in 1994 with the highest honor (Flicitations du Jury) and an "Ingénieur" degree from the Ecole des Mines de Paris in 1991. After earning his engineering degree, he worked in the central research laboratories of Thomson-CSF for three years, working on some problems of infrared image segmentation using mathematical morphology. He then joined a start-up company named Animation Science in 1995, as director of research and development. The technology of particle systems for computer graphics and scientific visualization, developed by the company under his technical leadership received several awards, including the "European Information Technology Prize 1997" awarded by the European Commission (Esprit programme) and by the European Council for Applied Science and Engineering and the "Hottest Products of the Year 1996" awarded by the Computer Graphics World journal. In 1998, he joined OCÉ Print Logic Technologies, as senior scientist. He worked there on various problem of image analysis dedicated to scanning and printing. In 2002, he joined the Informatics Department of ESIEE, Paris, where he is professor and a member of the Institut Gaspard Monge, Université Paris-Est Marne-la-Vallée. His current research interest is discrete mathematical morphology. 